\title{
The Plasmodium falciparum Drugome And Its Polypharmacological Implications
}

Yinliang Zhang' Li Xie $^{2}$, Lei Xie ${ }^{3,4}$, Philip E. Bourne ${ }^{5, *}$

1 School of Life Sciences, Anhui University, Hefei, Anhui, China.

2 Scripps Ranch, San Diego, CA, USA.

3 The Graduate Center, The City University of New York, New York, USA.

4 Department of Computer Science, Hunter College, The City University of New York, New York, USA.

5 Office of the Director, National Institutes of Health, Bethesda, MD, USA.

KEYWORDS: drug repurposing, antibacterial , malaria, drug-target network, structural proteome, systems pharmacology. 
Malaria is a disease contracted by over 200 million people each year, mostly in developing countries. The primary causative agent, Plasmodium falciparum (P.falciparum) has shown increased resistance to existing drugs, hence new treatments are needed quickly. To this end we performed a high-throughput systems-level analysis, mapping existing FDA drugs with the potential for repurposing against targets from the $P$. falciparum structural proteome. The resulting $P$. falciparum drugome (P.falciparum-drugome) was used to prioritize potential new anti-malaria candidate targets and highlight some novel FDA approved drugs that have apparent anti-malaria effects for possible use as multi-target therapeutics.

\section{INTRODUCTION}

The emerging field of systems pharmacology is changing the way we think about drugs. The traditional "one-drug one-target one-disease" paradigm has proven inadequate for drug discovery ${ }^{1}$; whereas the idea of multi-target drug interactions leading to the observed phenotype is now widely accepted ${ }^{2}$. Stated another way, a drug response is a consequence of complex interactions between multiple intracellular and extracellular components. Thus designing drugs assuming multiple targets is becoming the new rational approach to drug discovery, but requires a system level view of drug action. Systems pharmacology provides that view through a systematic understanding of drug action by integrating systems biology (including biological network analysis 3 ), bioinformatics and cheminformatics approaches. 
Simultaneously, network analysis approaches of a different kind have proven useful for organizing high-dimensional biological datasets and extracting meaningful information. Such network approaches in systems pharmacology can provide a global view of drug relationships, identify new drug targets as well as therapeutic strategies, and improve our understanding of the side effects and alternative uses of current drugs ${ }^{3-4}$. Here we exploit such a network approach in determining the P.falciparum drugome, a structural proteome-wide drug-target interaction network that forms a basis for exploring alternative treatments for malaria.

Malaria is one of the most devastating and widespread tropical parasitic diseases and is most prevalent in developing countries. The World Health Organization has estimated that over 200 million cases of malaria occur annually 5; in 2010, around 655,000 deaths were reported, but this is likely a significant underestimate. Approximately $87 \%$ of these deaths were children under the age of five ${ }^{6}$. To reduce the number of malaria infections and to reduce the death toll is an urgent priority.

Malaria is caused by the Plasmodium parasite, which is transmitted by a mosquito vector. In humans, the parasites multiply in the liver, and then infect red blood cells. There are five species that are known to infect humans: P.falciparum, Plasmodium vivax, Plasmodium ovalae, Plasmodium malariae and Plasmodium knowelsi. Among these five species, the parasite $P$. falciparum is the most dangerous, with the highest rates of complications and mortality. Currently, there are no approved vaccines available and given increasing drug resistance, finding novel anti-malarial drugs and associated targets appears the most efficient way to fight malaria ${ }^{7}$ 
Previously Gamo et al. ${ }^{8}$ screened nearly 2 million compounds from the GSK chemical library, of which more than 13 thousand were confirmed to inhibit P. falciparum growth. Jensen et al. 9 mapped the genome of P.falciparum to this drug-like chemical space using the chemical-protein annotation from the ChemProt database. However, establishing a new drug from such a large list of promising compounds is a lengthy, expensive and inefficient process ${ }^{10}$. As the pharmacologist and Nobel laureate James Black said, "the most fruitful basis for the discovery of a new drug is to start with an old drug" ${ }^{11}$. This was the approach taken by Kinnings et al. who reported a TBdrugome constructed from FDA-approved drugs and Mycobacterium tuberculosis (M.tb) proteins, and revealed that one-third of the FDA-approved drugs had the potential to be repositioned to treat tuberculosis and many currently unexploited M.tb receptors might be chemically druggable and could serve as novel anti-tubercular targets ${ }^{12}$.

In the spirit of Kinnings et al. ${ }^{12}$ the objective of the work described here is to provide a systems level view of malaria where all accessible putative target proteins are associated with all FDAapproved drugs. Hence, we built a structural proteome-wide drug-target network combining $P$. falciparum proteins with FDA-approved drugs, referred to as the P.falciparum drugome (P.falciparum-drugome). Using the P.falciparum-drugome we prioritized potential anti-malaria candidate targets and highlighted some novel drugs that have apparent anti-malaria effects as multi-target therapeutics. We regard the P.falciparum-drugome as a high-throughput approach to hypothesis generation, where the most promising theoretical drug target interactions can be tested experimentally. 


\section{RESULTS}

\section{A drug binding site database}

A total of 308 different drugs approved for human use were identified in the RCSB Protein Data Bank (PDB) ${ }^{13}$. Of these drugs, 135 were co-crystallized within a single protein structure, while the rest were co-crystallized with at least two protein structures (Figure 1), bringing the total number of drug binding sites in the PDB to 1423.

\section{The P.falciparum-drugome: A drug-protein network}

In the P.falciparum-drugome, 116 drugs are connected to 268 P. falciparum proteins (Figure 2). The Pfalciparum-drugome is a proteome-wide drug-protein interaction network, which consists of 384 nodes and 1120 edges (Figure 2). Each node represents either an FDA-approved drug or a P.falciparum protein; an edge is formed when a $P$. falciparum protein has a similar binding site to the known drug target as found in the PDB (see Methods). Of 308 drugs, 116 are connected to 268 P. falciparum proteins that originate from a total of 1569 P. falciparum solved structures or models.

We used SMAP ${ }^{14}$ to compare all PDB identified drug binding sites to all P. falciparum proteins with available structures and then subsequently excluded target-protein pairs whose global structures are significantly similar (see Methods). The average connectivity of the drugs was 
calculated in order to determine the p-value threshold for SMAP. As shown (Figure 3), an inflection point occurs at a p-value of $1.0 \mathrm{e}-5$, that is, when the p-value is less than $1.0 \mathrm{e}-5$, the drug connectivity only changes slightly, but it increases rapidly when the p-value is larger than 1.0e-5. Xie et al. previously reported that the false-positive rate is approximately $5 \%$ when the SMAP p-value is close to $1.0 \mathrm{e}-5^{14 \mathrm{~b}}$. The correlation between $\mathrm{p}$-value and drug degree observed here is consistent with that observed for the TB-drugome ${ }^{12}$ and hence a p-value $<1.0 \mathrm{e}-5$ is considered a good measure of true binding site similarity.

\section{The P.falciparum-drugome is scale-free}

The distribution of target connectivity follows a power-law distribution (Figure 4), indicating that the P.falciparum-drugome is a scale-free network rather than random. That is, a small number of $P$. falciparum proteins are connected to a large number of drugs while the majority of P. falciparum proteins have few connections. The TB-drugome is also a scale-free network ${ }^{12}$, suggesting that being scale-free is a common feature of these drugome networks and speaks to either a consistency in the variability of ligand binding sites across species, a limitation in the chemical space explored by existing FDA approved drugs, or both.

\section{Antimicrobial drugs are most likely to be anti-malarial drugs}

Given the distribution by species of FDA approved drugs bound to their primary targets as found in the PDB and the inferred targets in the P.falciparum-drugome, an interesting question is whether drugs targeting a specific species are favored against $P$. falciparum. Figure 5 illustrates 
the affected organisms based on drug binding as found in the PDB (Figure 5A) and drugs binding to proteins in the P.falciparum-drugome (Figure 5B), respectively. Based on information from DrugBank, 74\% of approved drugs target humans and other mammals; this number decreases to $62 \%$ in the P.falciparum-drugome. As expected, the distribution of drugs that primarily affected on bacteria, virus, fungi and protozoa increase from $11 \%, 3 \%, 3 \%$ and $3 \%$ to $13 \%, 13 \%, 7 \%$ and $5 \%$ respectively, indicating a higher propensity for antimicrobial drugs to bind to P. falciparum proteins.

Among all those antimicrobial drugs, the distribution of antiviral drugs increased most significantly, from $3 \%$ to $13 \%$ (Figure 5). Further investigation shows that 12 out of 16 antiviral drugs in P.falciparum-drugome are anti-HIV drugs. The association between HIV and malaria has always been an interesting issue, and many HIV protease inhibitors do have antimalarial activity $^{15}$. Several HIV-1 protease inhibitors are proved inhibit the growth of P. falciparum in vitro at clinically relevant concentrations while the mechanisms are poorly understood ${ }^{16}$. According to P.falciparum-drugome, the binding sites are similar between the targets of these antiviral drugs and P. falciparum proteins. This may explain why most anti-HIV drugs can also inhibit malaria, yet more studies are required to verify this hypothesis.

\section{Highly connected drugs are candidates for multi-target therapeutics}


The P.falciparum-drugome reveals that, of the 308 different drugs investigated, 88 drugs could potentially inhibit more than one P. falciparum protein. This is advantageous because multi-target therapeutics can be more efficacious and less vulnerable to adaptive resistance ${ }^{17}$. Highly connected drugs have the potential to inhibit a large number of different P. falciparum proteins simultaneously, a literature research of the highly connected drugs also support this hypothesis.

The top nine highly connected drugs are further revised. With 76 cross-fold connections, alitretinoin, a drug used to treat cutaneous lesions in patients with Kaposi's sarcoma, is the most highly connected drug. Alitretinoin, also called 9-cis-retinoic acid, is a metabolite of vitamin A. It is well known that supplementation with vitamin A can significantly reduce the malaria infection and decreases the mortality of infected young children ${ }^{18}$. With 70 cross-fold connections, methotrexate, an anti-cancer drug, is the second most highly connected drug. Note that although serum albumin is listed as one intended target of methotrexate, this drug has not actually been crystallized with serum albumin in PDB, so this does not account for its high connectivity. Several groups have shown that methotrexate is extremely effective against P. falciparum in vitro ${ }^{19}$. Nzila group has tested methotrexate in four murine species, however, none of them were susceptible to methotrexate. Noteworthy that they also tested the efficacy of pyrimethamine, a known antimalarial drug, in combination with folic acid in Plasmodium berghei, a murine plasmodium species that highly similar to human parasite $P$. falciparum, and data indicate that folic acid does not influence pyrimethamine efficacy, which suggests that the murine plasmodium, $P$. berghei, may not transport folate. Since methotrexate utilizes folate receptor/transport to gain access to cells, its lack of efficacy against the four tested murine 
malaria species may be the result of inefficiency of drug transport ${ }^{20}$. It is worth mentioning that a phase I trial in Kenyan adult healthy volunteers was carried out, and result suggests that lowdose methotrexate had an acceptable safety, but methotrexate blood levels did not reach the desirable concentration to clear malaria infection, further dose finding is necessary ${ }^{21}$. With 63 different cross-fold connections, levothyroxine, a drug used to treat hypothyroidism, is the third most highly connected drug. Further investigation revealed that it was the structure of levothyroxine bound in the binding site of serum albumin that was determined to be significantly similar to 44 of the 63 different $P$. falciparum binding sites. As a non-specific binder of steroid hormones and a transport protein for various fatty acids, serum albumin is known to be a highly promiscuous protein ${ }^{22}$. To our knowledge, there is no report of levothyroxine having any effect on malaria. Besides, the forth highly connected drug, raloxifene, a drug used to prevent and treat osteoporosis and breast cancer in women, also hasn't been reported affecting malaria. The fifth highly connected drug, estradiol, is a sex hormone. In the past few years, evidence has accumulated that many hormones, especially the sex steroids, can influence the immune system and thus susceptibility for diseases caused by protozoan parasites ${ }^{23}$. An in vivo experiment shows that estradiol can decreases parasitemia, but increases the incidence of cerebral malaria and the mortality in $P$. berghei infected mice 24 . Although the complete mechanism has not yet been unraveled, there is still an evidence that estradiol has effect on P. falciparum proteins.

It is worth to notice that drugs with an above-average number of connections are either antibacterial drugs or human affecting drugs (Figure 6). Although the whole distribution increased slightly, part of the antibacterial drugs are connected to a larger number of different $P$. 
falciparum proteins than antiviral drugs, making these certain antibacterial drugs the most likely candidates against malaria assuming the goal is to impact as many targets as possible in the battle to overcome resistance through mutation.

The sixth highly connected drug, fusidic acid, is a bacteriostatic antibiotic. Many studies suggest that fusidic acid has potential activity against malaria ${ }^{25}$, Johnson et al. shows that fusidic acid inhibited the growth of P. falciparum erythrocytic stages with immediate death effect ${ }^{26}$. Other antibacterial drugs whose connections are above average are rifampin, trimethoprim and tetracycline. The antimalarial activity of rifampin was confirmed with P. falciparum in vitro ${ }^{27}$, and a combination with other 2 drugs was verified safe and efficacious for treating malaria in murine model ${ }^{28}$. Trimethoprim is a pyrimidine inhibitor of dihydrofolate reductase, and World Health Organization (WHO) guidelines for the treatment of malaria recommend a combination of trimethoprim and sulfamethoxazole as prophylaxis for HIV-infected patients to protect against malaria ${ }^{29}$. Tetracycline is a broad spectrum antibiotic, and has also been recommended by WHO as second-line antimalarial treatment when combined with artesunate or quinie ${ }^{29}$.

To further investigate the effects of gene encoding P. falciparum proteins in metabolism, we here use a genome-scale flux-balanced metabolic network model ${ }^{30}$. We revised top 9 highly connected drugs and the P. falciparum proteins they connected to which are predicted essential for growth in the unconstrained metabolic network of P.falciparum. All of these 9 drugs are predicted bind 
at least one essential P.falciparum protein, indicating the highly connected drugs have high potential to be antimalarial drugs.

The concept of "synthetic lethality" - some genes may not essential on their own, but are lethal if deleted simultaneously - sheds new light on drug development ${ }^{31}$. The most interesting thing is that, in the P.falciparum metabolic network, they predict 16 gene pairs that have synthetic lethality, and one of them occurs in alitretinoin, levothyroxine and mifepristone. Another pair of the synthetic lethal genes is connected to different drugs, making it possible to use these drugs as combination therapy.

According to these experimental supports, it is highly believed that with the help of our discovery the representative drugs listed in our P.falciparum-drugome are worth exploring and testing thoroughly.

\section{DISCUSSION}

Existing drug-target networks are typically constructed from either annotated drug-target pairs or transference of chemical properties. Here we present our second (the first was the TB-drugome) high throughput structural-based proteome-wide drug-target network, that of P. falciparum - the P.falciparum-drugome. By using Food and Drug Administration (FDA) approved drugs we present the raw data for possible repurposing strategies, since these drugs are assumed safe. By 
presenting the drug target interaction network based on binding site similarity across much of the P. falciparum proteome we provide raw data on possible new targets for these drugs where the hubs represent the best opportunities to combat resistance. Experimental support exists for some of our theoretical findings and a variety of putative new targets and repurposing opportunities are presented in the supplemental materials. Simultaneously we are beginning to examine the TBand $P$. falciparum drugomes against the emergent human drugome. This will be the subject of a future publication. Likewise, we are working on a workflow software system that will enable others to simply compute drugomes or update existing drugomes as new drugs and putative targets become available.

It is worth mentioning that Yuan et al used a high-throughput chemical screening approach, and identified 32 highly active compounds ${ }^{32}$. Medicines for Malaria Ventrue (MMV) also provides a resource named Malaria Box, which contains 200 drug-like compounds and 200 probe-like compounds, and they are selected by in vitro screening against P. falciparum 3D7 from 20,000 compounds in GlaxoSmithKline Tres Cantos Antimalarial Set (TCAMS), Novartis-GNF dataset and St. Jude Children's Research Hospital's dataset ${ }^{33}$. The compounds they provide are different from the drugs in our drugome, mainly because most of the compounds are not approved drugs or have no co-crystallized structure as yet. While chemical high throughput screening will find compounds with direct antimalarial effect, some of the most promising candidates for combined therapy may be missed. For example, the highly connected drug in our drugome, rifampin, which is proven safe and efficacious for treating malaria in a murine model when combined with isoniazide and ethambutol, but not alone ${ }^{28}$, does not exist in either Yuan's work or Malaria Box. 
Notwithstanding there are some unavoidable limitations to our approach. First, although the genome of $P$. falciparum is sequenced ${ }^{34}$, structural coverage is only $2.1 \%$ from experimental structure and $12.5 \%$ when homology models are included. Models may be of limited accuracy, being modeled from single chains, missing many protein-drug interactions that occur at the interfaces between tertiary units of the protein and only observed if the quaternary structure is modeled. Even more limiting is the bias in the PDB from which experimental structures are drawn and models inferred. Many drug targets, notably membrane proteins, are underrepresented in the PDB. While the number of FDA approved drugs rises slowly the structural coverage of a variety of proteomes, including human is increasing more rapidly and will only see this type of high throughput technique improve, especially when used with emergent methods from systems pharmacology.

\section{MATERIALS AND METHODS}

\section{Structural coverage of the $P$. falciparum proteome}

There are 5491 protein-encoding genes in the P.falciparum proteome ${ }^{34}, 118$ of which have solved structures available in the RCSB Protein Data Bank ${ }^{13}$ (Oct 18, 2011). A single protein sequence may have several associated structures co-crystallized with different ligands, or containing different point mutations. All are important in comparing binding sites, which brings the total number of known P. falciparum protein structures to 333 (Oct 18, 2011). 
To maximize coverage of the P. falciparum proteome we also include homology models deposited in ModBase ${ }^{35}$, a database of annotated comparative protein structure models and associated resources. This database contains over 20 thousands homology models for the entire P. falciparum proteome since ModBase has several models for each protein sequence; a model is considered reliable if its Model score (GA341) is greater than 0.7 and its ModPipe Protein Quality Score (MPQS) is greater than 1.1 (http://modbase.compbio.ucsf.edu/modbase-cgi/ display.cgi?type=help\&server=modbase). Employing these thresholds, there are 1236 reliable homology models (Oct 18, 2011), and the total structural coverage of the P. falciparum proteome is $12.5 \%$. As indicated in the Discussion, only a single chain of each homology model is available, rather than the entire biological unit.

\section{Identification of FDA-approved drug binding sites}

The DrugBank database ${ }^{36}$ combines detailed drug and target information where known (http:// www.drugbank.ca/). Drugs approved by the U.S. Food and Drug Administration (FDA) are taken from DrugBank and all the nutraceuticals removed. We use InChI keys to map these FDAapproved drugs to ligands in the PDB to find the associated co-crystalized structures. After removing non-protein structures, such as DNA, RNA and associated complexes, there are 308 FDA-approved drugs with 1423 drug-protein binding sites (Dec 6, 2011).

\section{Comparison of ligand binding sites using SMAP}


Xie et al. developed SMAP ${ }^{14}$ for the comparison of potential protein three-dimensional ligand binding site motifs in a manner that is independent of the sequence order. Proteins are represented by their shapes, as defined by using only $\mathrm{C} \alpha$ atoms. Then ligand binding sites of the two proteins are aligned and assigned a p-value measuring their similarity based on a unified statistical model ${ }^{14 b}$.

We compare 1423 drug binding sites against 1569 P. falciparum proteins, including both experimental structures and homology models. The entire structure of each $P$. falciparum protein is used to avoid missing alternative binding sites on any given $P$. falciparum protein. A $p$-value is reported to measure the similarity of the binding sites for each pair.

\section{Comparison of global protein structures using FATCAT}

FATCAT (Flexible structure AlignmenT by Chaining Aligned fragment pairs allowing Twists) ${ }^{37}$ is an approach for flexible protein structure comparison. The Java version of FATCAT was downloaded from the RCSB PDB website and run locally (http://pdbx.org/jfatcatserver/ download.jsp). 
In order to detect only remote similarities between the P. falciparum proteins and drug binding site proteins, that is, those which cannot be identified through global structure comparison, we exclude those pairs identified by FATCAT where the p-value was less than 0.5 .

\section{Visualization of the protein-drug interaction network}

We use yEd from yWorks to visualize the protein-drug interaction network (http:// www.yworks.com/en/products_yed_about.html). A node represents a P.falciparum structure or a drug ligand. If a P. falciparum structure and a drug binding site have similar binding sites, and their global structures are dissimilar (according to the criteria outlined above), we place an edge between the $P$. falciparum structure and the associated drug.

\section{GO term enrichment analysis}

To evaluate the functional relationships between target proteins of each highly connected drug, we use DAVID (Database for Annotation, Visualization and Integrated Discovery) ${ }^{38}$ to perform a GO term enrichment, since DAVID provides a comprehensive set of functional annotation tools for investigators to understand biological meaning behind large list of genes. DAVID uses a modified Fisher Exact p-value to measure the enrichment of each GO term. The smaller a $p$ value is, the more enriched this GO term is. 
We use the UniProt accessions of each target protein and associated drug binding sites to do the GO term enrichment. For each highly connected drug, we establish the GO term enrichment of the associated $P$.falciparum structures. In general terms, if these targets are in diverse pathways, this drug may hold promise for a multi-target treatment regime, with particular emphasis on avoiding resistance. Alternatively, if these targets are enriched in a specific pathway, this means the drug have the potential to treat the specific disease without side effects.

\section{FIGURES}

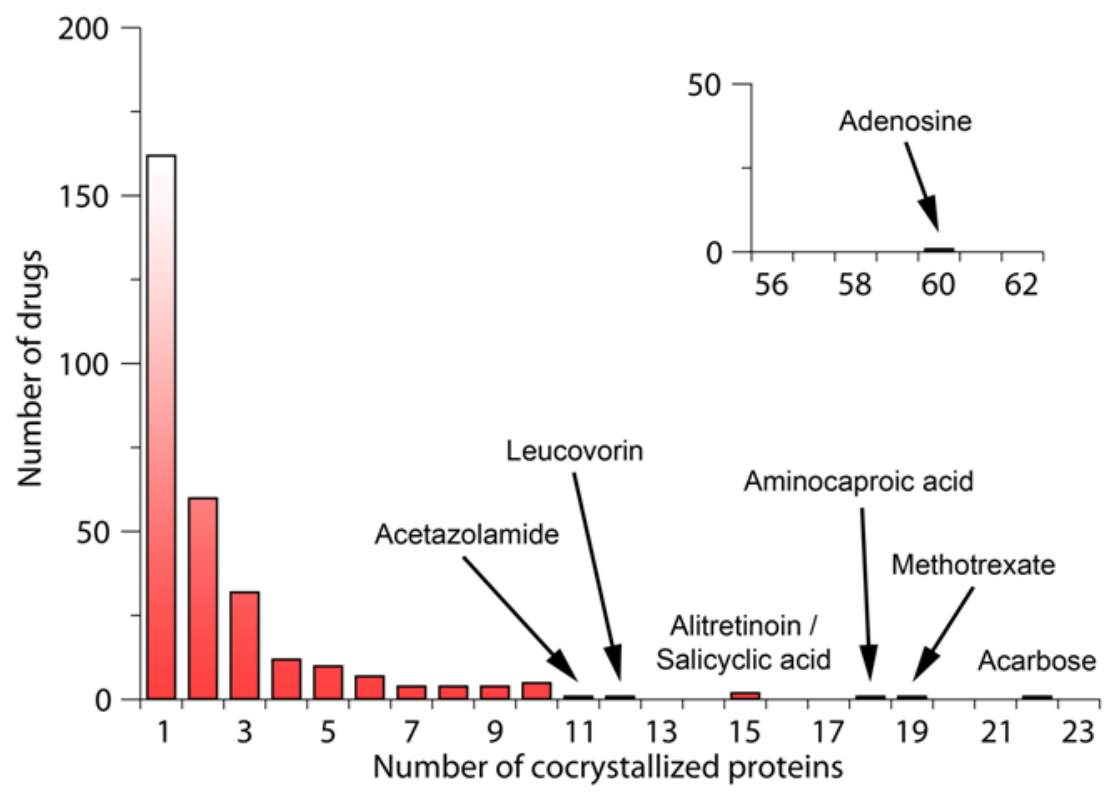

Figure 1. The numbers of unique proteins co-crystallized with approved drugs in the PDB. 


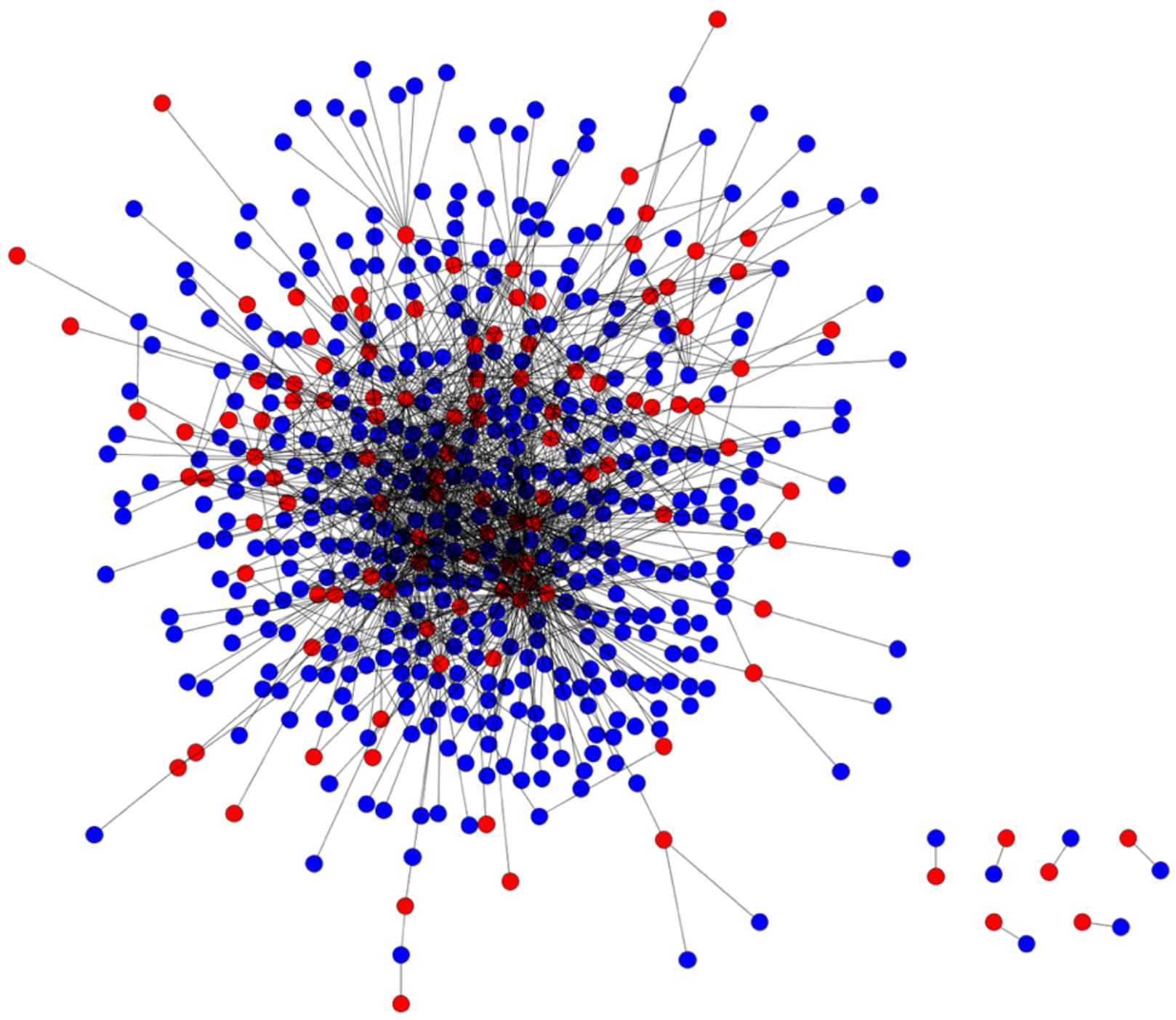

Figure 2. Protein-drug interaction network. The similarities between the binding sites of $P$.

falciparum proteins (blue), and binding sites containing approved drugs (red) were illustrated. An SMAP p-value threshold of 1.0e-5 was used to define similarity. 


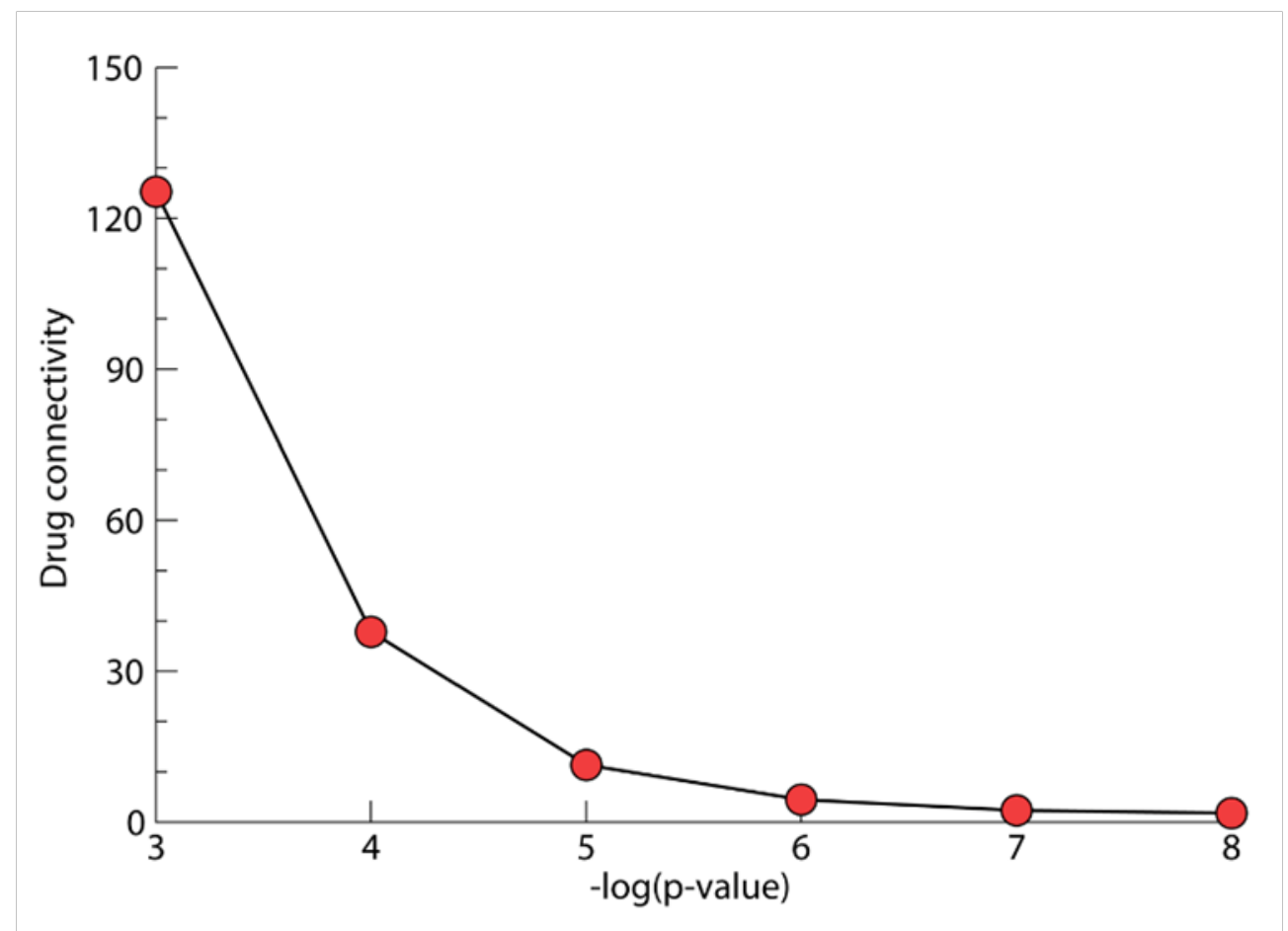

Figure 3. The average number of connections per drug in the P.falciparum-drugome plotted against the SMAP p-value threshold.

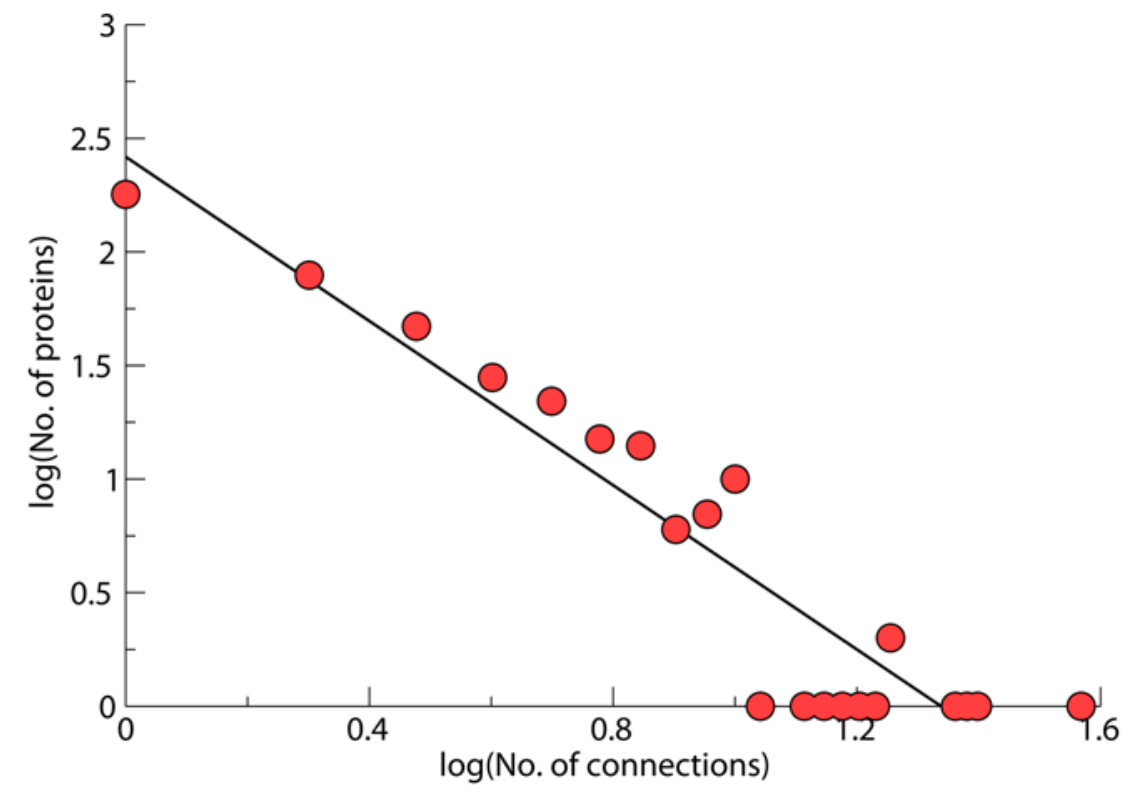


Figure 4. Fitting of the distribution of target connections to a power-law distribution for the P.falciparum-drugome. An SMAP p-value threshold of 1.0e-5 was used.
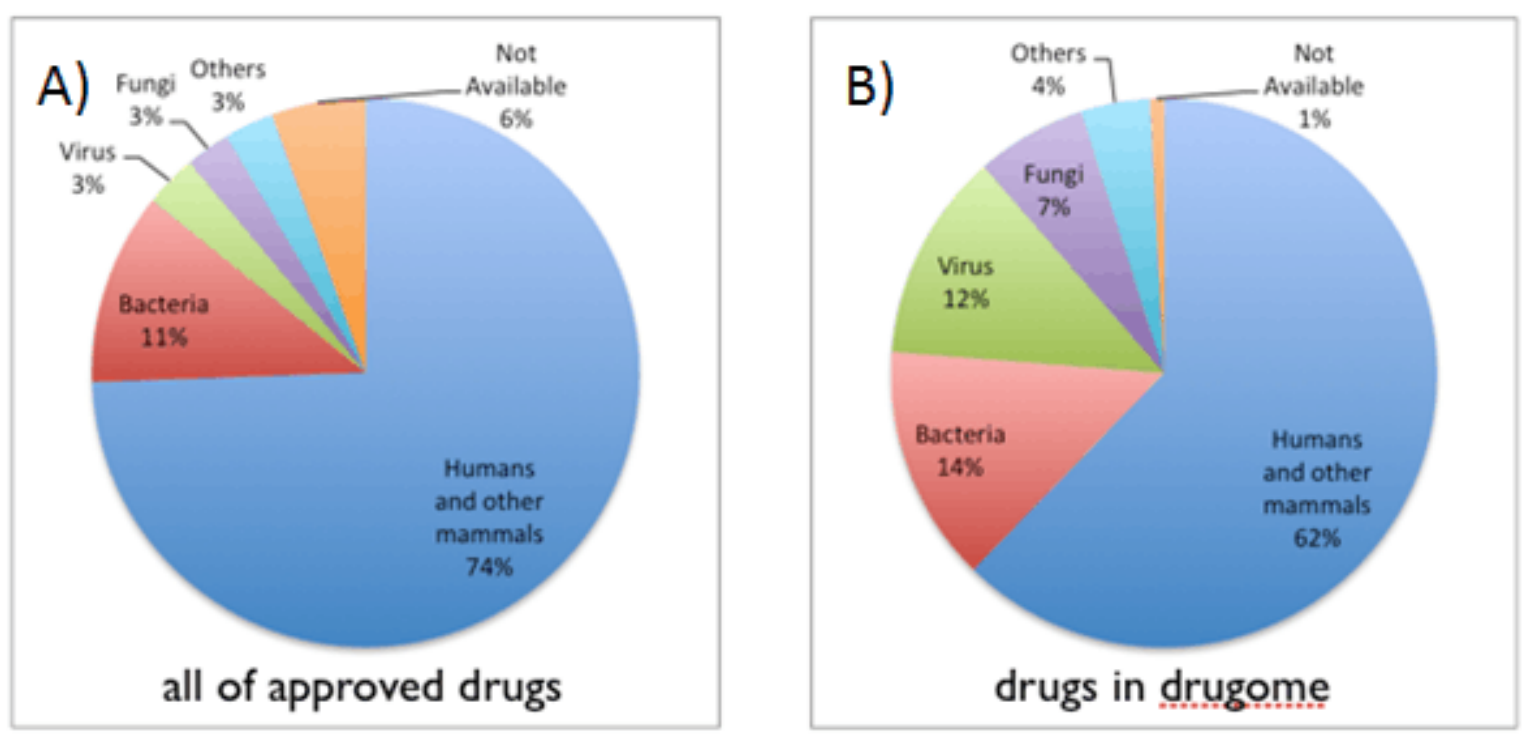

Figure 5. Drug distribution based on affected organisms. A) All approved FDA drugs. B)

Drugs forming the P.falciparum-drugome. 


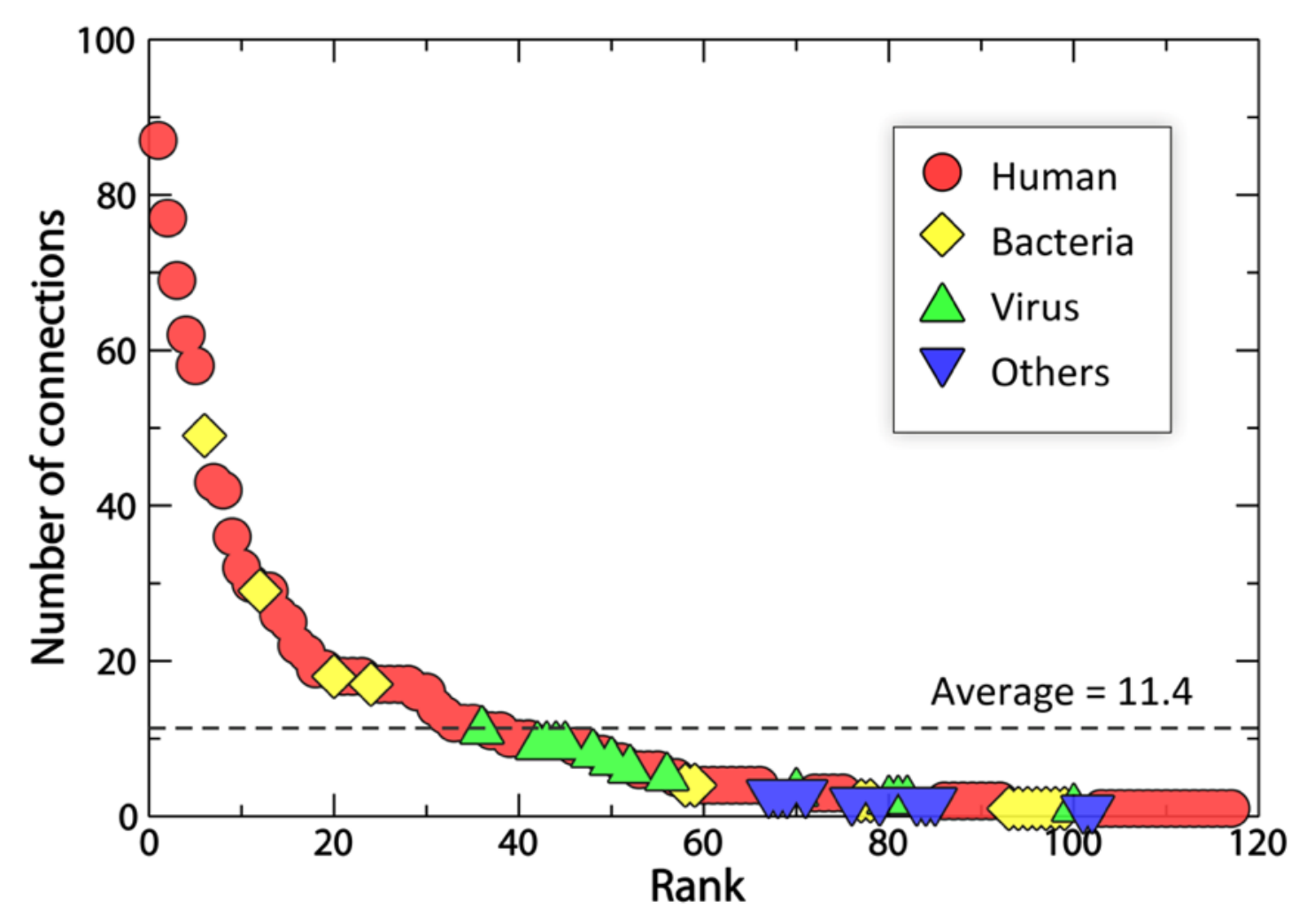

Figure 6. A ranking of FDA approved drugs according to the number of targets to which the drug is assumed to bind.

\section{Supporting Information}

Table S1. Information about the approved drug binding sites used in the P.falciparum-drugome. This file contains information about the 308 approved drugs that were identified in the PDB. For each drug, its name, PDB ligand code, isomeric SMILES string and known targets are listed, and the PDB codes of the protein structures with which it has been crystallized are given. 
Table S2. Cross-fold drug-target pairs in the P.falciparum-drugome (for solved P. falciparum structures only). This file contains a list of the cross-fold drug-target pairs with a SMAP P-value $<1.0 \mathrm{e}-5$, for solved P. falciparum structures only. For each pair, information about the drug and target structures is given, as well as the corresponding SMAP P-value (indicating the significance of the binding site similarity) and AutoDock Vina score (from docking the drug into the predicted binding site in the $\mathrm{P}$. falciparum protein).

Table S3. Cross-fold drug-target pairs in the P.falciparum-drugome (for P. falciparum homology models only). This file contains a list of the cross-fold drug-target pairs with a SMAP P-value $<1.0 \mathrm{e}-5$, for homology models of P. falciparum proteins only. For each pair, information about the drug and target structures is given, as well as the corresponding SMAP P-value (indicating the significance of the binding site similarity) and AutoDock Vina score (from docking the drug into the predicted binding site in the M.tb protein).

Table S4. Information about the solved P. falciparum structures used in the P.falciparumdrugome. This file contains information about the P. falciparum proteins with solved structure(s) in the RCSB PDB that were used in the P.falciparum-drugome. For each protein, the gene name, protein name and corresponding PDB codes are given.

Table S5. Information about the P. falciparum homology models used in the P.falciparumdrugome. This file contains information about the reliable homology models of P. falciparum 
proteins from ModBase that were used in P.falciparum-drugome. For each homology model, the ModBase model code is given, as well as the gene name and description of the P. falciparum protein.

\section{AUTHOR INFORMATION}

\section{Corresponding Author}

* To whom correspondence should be addressed philip.bourne@nih.gov

\section{ACKNOWLEDGMENT}

We thank Dr. Haiyan Liu for his helpful discussions and suggestions. Financial supports from China Scholarship Council are gratefully acknowledged.

\section{REFERENCES}

1. Xie, L.; Evangelidis, T.; Xie, L.; Bourne, P. E., Drug Discovery Using Chemical Systems

Biology: Weak Inhibition of Multiple Kinases May Contribute to the Anti-Cancer Effect of Nelfinavir. Plos Comput Biol 2011,7 (4). 
2. Xie, L.; Kinnings, S. L.; Bourne, P. E., Novel computational approaches to polypharmacology as a means to define responses to individual drugs. Annu Rev Pharmacol Toxicol 2012,52, 361-79.

3. (a) Berger, S. I.; Iyengar, R., Network analyses in systems pharmacology. Bioinformatics 2009,25 (19), 2466-2472; (b) Arrell, D. K.; Terzic, A., Network systems biology for drug discovery. Clin Pharmacol Ther 2010,88 (1), 120-5.

4. Yildirim, M. A.; Goh, K. I.; Cusick, M. E.; Barabasi, A. L.; Vidal, M., Drug-target network. Nat Biotechnol 2007,25 (10), 1119-26.

5. WHO, World Malaria Report 2008. 2008.

6. WHO, World Malaria Report 2011. 2011.

7. (a) WHO I Malaria

http://www.who.int/topics/malaria/en/;(b) P. falciparum $\underline{\text { http://en.wikipedia.org/wiki/ }}$ Plasmodium falciparum;(c) Burrows, J. N.; Waterson, D., Discovering New Medicines to Control and Eradicate Malaria. Elliott, R., Ed. Springer Berlin Heidelberg: 2011; Vol. 7, pp 125-180.

8. Gamo, F. J.; Sanz, L. M.; Vidal, J.; de Cozar, C.; Alvarez, E.; Lavandera, J. L.; Vanderwall, D. E.; Green, D. V. S.; Kumar, V.; Hasan, S.; Brown, J. R.; Peishoff, C. E.; Cardon, L. R.; Garcia-Bustos, J. F., Thousands of chemical starting points for antimalarial lead identification. Nature 2010,465 (7296), 305-U56.

9. Jensen, K.; Plichta, D.; Panagiotou, G.; Kouskoumvekaki, I., Mapping the genome of Plasmodium falciparum on the drug-like chemical space reveals novel anti-malarial targets and potential drug leads. Mol Biosyst 2012.

10. Drug Discovery http://en.wikipedia.org/wiki/Drug discovery. 
11. Chong, C. R.; Sullivan, D. J., New uses for old drugs. Nature 2007,448 (7154), 645-646.

12. Kinnings, S. L.; Xie, L.; Fung, K. H.; Jackson, R. M.; Xie, L.; Bourne, P. E., The Mycobacterium tuberculosis Drugome and Its Polypharmacological Implications. Plos Comput Biol 2010,6 (11).

13. Berman, H. M.; Westbrook, J.; Feng, Z.; Gilliland, G.; Bhat, T. N.; Weissig, H.; Shindyalov, I. N.; Bourne, P. E., The Protein Data Bank. Nucleic Acids Res 2000,28 (1), 235-42.

14. (a) Xie, L.; Bourne, P. E., A robust and efficient algorithm for the shape description of protein structures and its application in predicting ligand binding sites. Bmc Bioinformatics 2007,8;(b) Xie, L.; Bourne, P. E., Detecting evolutionary relationships across existing fold space, using sequence order-independent profile-profile alignments. P Natl Acad Sci USA 2008,105 (14), 5441-5446;(c) Xie, L.; Xie, L.; Bourne, P. E., A unified statistical model to support local sequence order independent similarity searching for ligand-binding sites and its application to genome-based drug discovery. Bioinformatics 2009,25 (12), I305-I312.

15. Slutsker, L.; Marston, B. J., HIV and malaria: interactions and implications. Curr Opin Infect Dis 2007,20 (1), 3-10.

16. (a) Skinner-Adams, T. S.; McCarthy, J. S.; Gardiner, D. L.; Hilton, P. M.; Andrews, K. T., Antiretrovirals as antimalarial agents. Journal of Infectious Diseases 2004,190 (11), 1998-2000; (b) Parikh, S.; Gut, J.; Istvan, E.; Goldberg, D. E.; Havlir, D. V.; Rosenthal, P. J., Antimalarial activity of human immunodeficiency virus type 1 protease inhibitors. Antimicrob Agents Ch 2005,49 (7), 2983-2985;(c) Martin, R. E.; Butterworth, A. S.; Gardiner, D. L.; Kirk, K.; McCarthy, J. S.; Skinner-Adams, T. S., Saquinavir Inhibits the Malaria Parasite's Chloroquine Resistance Transporter. Antimicrob Agents Ch 2012,56 (5), 2283-2289;(d) Nsanzabana, C.; 
Rosenthal, P. J., In Vitro Activity of Antiretroviral Drugs against Plasmodium falciparum. Antimicrob Agents Ch 2011,55 (11), 5073-5077.

17. Zimmermann, G. R.; Lehar, J.; Keith, C. T., Multi-target therapeutics: when the whole is greater than the sum of the parts. Drug Discovery Today 2007,12 (1-2), 34-42.

18. (a) Shankar, A. H.; Genton, B.; Semba, R. D.; Baisor, M.; Paino, J.; Tamja, S.; Adiguma, T.; Wu, L.; Rare, L.; Tielsch, J. M.; Alpers, M. P.; West, K. P., Effect of vitamin A supplementation on morbidity due to Plasmodium falciparum in young children in Papua New Guinea: a randomised trial. Lancet 1999,354 (9174), 203-209;(b) Serghides, L.; Kain, K. C., Mechanism of protection induced by vitamin A in falciparum malaria. Lancet 2002,359 (9315), 1404-1406;(c) Villamor, E.; Fawzi, W. W., Effects of vitamin A supplementation on immune responses and correlation with clinical outcomes. Clin Microbiol Rev 2005,18 (3), 446-+.

19. (a) Dar, O.; Khan, M. S.; Adagu, I., The potential use of methotrexate in the treatment of falciparum malaria: in vitro assays against sensitive and multidrug-resistant falciparum strains. Jpn J Infect Dis 2008,61 (3), 210-211;(b) Nduati, E.; Diriye, A.; Ommeh, S .; Mwai, L.; Kiara, S.; Masseno, V.; Kokwaro, G.; Nzila, A., Effect of folate derivatives on the activity of antifolate drugs used against malaria and cancer. Parasitol Res 2008,102 (6), 1227-1234;(c) Kiara, S. M.; Okombo, J.; Masseno, V.; Mwai, L.; Ochola, I.; Borrmann, S.; Nzila, A., In Vitro Activity of Antifolate and Polymorphism in Dihydrofolate Reductase of Plasmodium falciparum Isolates from the Kenyan Coast: Emergence of Parasites with Ile-164-Leu Mutation. Antimicrob Agents Ch 2009,53 (9), 3793-3798.

20. Irungu, B.; Kiboi, D.; Langat, B.; Rukunga, G.; Wittlin, S.; Nzila, A., Methotrexate and aminopterin lack in vivo antimalarial activity against murine malaria species. Exp Parasitol 2009,123 (2), 118-121. 
21. Chilengi, R.; Juma, R.; Abdallah, A. M.; Bashraheil, M.; Lodenyo, H.; Nyakundi, P.; Anabwani, E.; Salim, A.; Mwambingu, G.; Wenwa, E.; Jemutai, J.; Kipkeu, C.; Oyoo, G. O.; Muchohi, S. N.; Kokwaro, G.; Niehues, T.; Lang, T. D.; Nzila, A., A phase I trial to evaluate the safety and pharmacokinetics of low-dose methotrexate as an anti-malarial drug in Kenyan adult healthy volunteers. Malaria $J$ 2011,10.

22. Ghuman, J.; Zunszain, P. A.; Petitpas, I.; Bhattacharya, A. A.; Otagiri, M.; Curry, S., Structural basis of the drug-binding specificity of human serum albumin. J Mol Biol 2005,353 (1), 38-52.

23. Roberts, C. W.; Walker, W.; Alexander, J., Sex-associated hormones and immunity to protozoan parasites. Clin Microbiol Rev 2001,14 (3), 476-+.

24. Libonati, R. M. F.; Cunha, M. G.; Souza, J. M.; Santos, M. V. N.; Oliveira, S. G.; DanielRibeiro, C. T.; Carvalho, L. J. M.; do Nascimento, J. L. M., Estradiol, but not dehydroepiandrosterone, decreases parasitemia and increases the incidence of cerebral malaria and the mortality in Plasmodium berghei ANKA-infected CBA mice. Neuroimmunomodulat 2006,13 (1), 28-35.

25. (a) Black, F. T.; Wildfang, I. L.; Borgbjerg, K., Activity of Fusidic Acid against Plasmodium Flaciparum Invitro. Lancet 1985,1 (8428), 578-579;(b) Collignon, P.; Turnidge, J., Fusidic acid in vitro activity. Int J Antimicrob Ag 1999,12, S45-S58.

26. Johnson, R. A.; McFadden, G. I.; Goodman, C. D., Characterization of Two Malaria Parasite Organelle Translation Elongation Factor G Proteins: The Likely Targets of the AntiMalarial Fusidic Acid. PLoS One 2011,6 (6). 
27. Strath, M.; Scott-Finnigan, T.; Gardner, M.; Williamson, D.; Wilson, I., Antimalarial activity of rifampicin in vitro and in rodent models. Trans $R$ Soc Trop Med Hyg 1993,87 (2), 211-6.

28. Aditya, N. P.; Patankar, S.; Madhusudhan, B., Assessment of in vivo antimalarial activity of rifampicin, isoniazide, and ethambutol combination therapy. Parasitol Res 2010,106 (6), $1481-1484$.

29. WHO, Guidelines for the treatment of malaria, second edition. 2010.

30. Plata, G.; Hsiao, T. L.; Olszewski, K. L.; Llinas, M.; Vitkup, D., Reconstruction and fluxbalance analysis of the Plasmodium falciparum metabolic network. Molecular Systems Biology 2010,6 .

31. (a) Tucker, C. L.; Fields, S., Lethal combinations. Nature Genet. 2003,35 (3), 204-205;(b)

Hopkins, A. L., Network pharmacology: the next paradigm in drug discovery. Nat Chem Biol 2008,4 (11), 682-690.

32. Yuan, J.; Cheng, K. C. C.; Johnson, R. L.; Huang, R. L.; Pattaradilokrat, S.; Liu, A. N.; Guha, R.; Fidock, D. A.; Inglese, J.; Wellems, T. E.; Austin, C. P.; Su, X. Z., Chemical Genomic Profiling for Antimalarial Therapies, Response Signatures, and Molecular Targets. Science 2011,333 (6043), 724-729.

33. Duffy, S.; Avery, V. M., Development and Optimization of a Novel 384-Well AntiMalarial Imaging Assay Validated for High-Throughput Screening. Am J Trop Med Hyg 2012,86 (1), 84-92.

34. Gardner, M. J.; Hall, N.; Fung, E.; White, O.; Berriman, M.; Hyman, R. W.; Carlton, J. M.; Pain, A.; Nelson, K. E.; Bowman, S.; Paulsen, I. T.; James, K.; Eisen, J. A.; Rutherford, K.; Salzberg, S. L.; Craig, A.; Kyes, S.; Chan, M. S.; Nene, V.; Shallom, S. J.; Suh, B.; Peterson, J.; 
Angiuoli, S.; Pertea, M.; Allen, J.; Selengut, J.; Haft, D.; Mather, M. W.; Vaidya, A. B.; Martin, D. M. A.; Fairlamb, A. H.; Fraunholz, M. J.; Roos, D. S.; Ralph, S. A.; McFadden, G. I.; Cummings, L. M.; Subramanian, G. M.; Mungall, C.; Venter, J. C.; Carucci, D. J.; Hoffman, S. L.; Newbold, C.; Davis, R. W.; Fraser, C. M.; Barrell, B., Genome sequence of the human malaria parasite Plasmodium falciparum. Nature 2002,419 (6906), 498-511.

35. Pieper, U.; Webb, B. M.; Barkan, D. T.; Schneidman-Duhovny, D.; Schlessinger, A.; Braberg, H.; Yang, Z.; Meng, E. C.; Pettersen, E. F.; Huang, C. C.; Datta, R. S.; Sampathkumar, P.; Madhusudhan, M. S.; Sjolander, K.; Ferrin, T. E.; Burley, S. K.; Sali, A., ModBase, a database of annotated comparative protein structure models, and associated resources. Nucleic Acids Res 2011,39 (Database issue), D465-74.

36. Knox, C.; Law, V.; Jewison, T.; Liu, P.; Ly, S.; Frolkis, A.; Pon, A.; Banco, K.; Mak, C.; Neveu, V.; Djoumbou, Y.; Eisner, R.; Guo, A. C.; Wishart, D. S., DrugBank 3.0: a comprehensive resource for 'omics' research on drugs. Nucleic Acids Res 2011,39 (Database issue), D1035-41.

37. Ye, Y.; Godzik, A., Flexible structure alignment by chaining aligned fragment pairs allowing twists. Bioinformatics 2003,19 Suppl 2, ii246-55.

38. Huang, D. W.; Sherman, B. T.; Lempicki, R. A., Systematic and integrative analysis of large gene lists using DAVID bioinformatics resources. Nat Protoc 2009,4 (1), 44-57. 